\title{
Does the level of serum antimüllerian hormone predict ovulatory function in women with polycystic ovary syndrome with aging?
}

\author{
Enrico Carmina, M.D., ${ }^{a}$ Anna Maria Campagna, M.D., ${ }^{a}$ Pasquale Mansuet, M.D., ${ }^{b}$ Giustina Vitale, M.D., \\ Daniel Kort, M.D., ${ }^{c}$ and Roger Lobo, M.D. ${ }^{c}$ \\ ${ }^{a}$ Department of Medical and Biological Sciences and ${ }^{\mathrm{b}}$ Department of Clinical Medicine, University of Palermo, Palermo, \\ Italy; and ' Department of Obstetrics and Gynecology, Columbia University, New York, New York
}

Objective: To determine possible prediction of regular menses with aging in anovulatory women with polycystic ovary syndrome (PCOS).

Design: Cohort.

Setting: Academic practice.

Patient(s): A total of 54 anovulatory women with PCOS and 28 age- and weight-matched control subjects.

Intervention(s): Blood and ovarian ultrasound at baseline and after 5 years.

Major Outcome Measure(s): Serum antimüllerian hormone (AMH), gonadotropins, androgens, insulin sensitivity, and ovarian ultrasound.

Result(s): After 5 years, there was a significant decrease in AMH in women with PCOS and control subjects (10 of 54 anovulatory women became ovulatory after 5 years). There was a significant negative correlation between baseline serum AMH and ovulatory function after 5 years. However, baseline serum $\mathrm{AMH} \leq 4 \mathrm{ng} / \mathrm{mL}$ was associated with ovulatory function.

Conclusion(s): Serum AMH may help predict ovulatory function with aging in anovulatory women with PCOS. (Fertil Steril ${ }^{\circledR}$ 2012;98:1043-6. (C)2012 by American Society for Reproductive Medicine.)

Key Words: PCOS, ovulation, AMA, aging

Discuss: You can discuss this article with its authors and with other ASRM members at http:// fertstertforum.com/carminae-amh-ovulatory-function-polycystic-ovary-syndrome-aging/

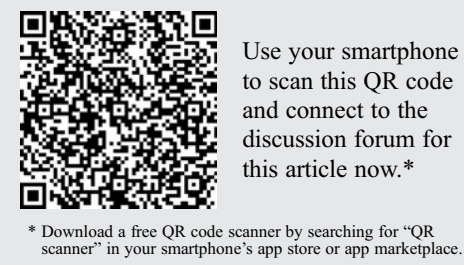
to scan this QR code connect to the discussion forum for

0 everal studies have suggested that many of the symptoms and signs of polycystic ovary syndrome (PCOS) may improve with aging (1-4). Indeed, a proportion of women with PCOS, previously with irregular menses, may become more regular and ovulate during late adulthood. In our long-term longitudinal study of 193 women with PCOS, about 30\% of women had improved symptoms and signs by their mid-40s, and $\sim 10 \%$ could no longer be diagnosed as having PCOS by established criteria. Another $20 \%$ of women had normal menses and ovulatory function, but still met the criteria for the diagnosis of PCOS by the Rotterdam criteria (4).

These findings are at least in part due to the decline in ovarian and adrenal androgens that occur in both normal women and those with PCOS as

Received March 14, 2012; revised May 15, 2012; accepted June 7, 2012; published online July 6, 2012 E.C. has nothing to disclose. A.M.C. has nothing to disclose. P.M. has nothing to disclose. G.V. has nothing to disclose. D.K. has nothing to disclose. R.L. has nothing to disclose.

Reprint requests: Roger Lobo, M.D., Columbia University Medical Center, 622 West, 168 Street Rm. 16-65, New York, NY 10032 (E-mail: ral35@columbia.edu).

they move from the third to the fifth decade of life. In normal women, ovarian androgen responses to hCG begin to decline at the age of 30 years (5) and a marked decrease in adrenal androgen secretion takes place between the second and fifth decades (6). Also, ovarian size has been shown to decrease with aging $(7,8)$.

In PCOS, some studies have shown that both total and non-SHBG-bound testosterone is 50\% lower at $45-47$ years compared with the levels found in patients in their 20s and 30s (9). Similarly, older patients with PCOS have lower adrenal androgen levels and smaller ovaries compared with young women with PCOS $(2,9,10)$. In our longitudinal study, a decrease of 
testosterone levels of $\sim 25 \%$ and of DHEAS of $\sim 30 \%$ was observed in 193 women with PCOS who were followed from a mean age of 22 years to a mean age of 43 years (4). Ovarian size was also reduced, with the mean ovarian size decreasing by $\sim 20 \%$ (from 10.9 to $9.1 \mathrm{~mL}$ ) (4).

Previous work has suggested that the regularity of menstrual cycles with aging in women with PCOS may be related to a smaller follicle cohort size in those women (11). However, it has not been determined if any parameters may be useful in helping to predict those women who will attain regular cycles among the general population of women with PCOS who have irregular cycles. Here we sought to determine if any parameters may be helpful in this regard in a prospective cohort of women with PCOS followed for 5 years. We focused our attention on changes in levels of serum antimüllerian hormone (AMH), because it relates well to the size of the total follicular cohort of the ovary.

\section{MATERIALS AND METHODS}

In this prospective study, 54 anovulatory hyperandrogenic women with PCOS aged 35-39 years (mean $37 \pm 1$ years) were evaluated. The diagnosis of PCOS was made according to Rotterdam criteria (12). The women had been treated previously with various therapies for menstrual irregularity (oligomenorrhea or amenorrhea) and/or hyperandrogenism (acne or hirsutism) but had not received any treatment for $\geq 3$ months before evaluation in this study. Menstrual cycles were recorded for $\geq 3$ months and oligomenorrhea was defined as menstrual cycle intervals $>35$ days.

Twenty weight-matched normal ovulatory women of similar age (mean age $37 \pm 1$ years) also were evaluated initially and again after 5 years. Normal menses were defined as cycles lasting 25-34 days.

In both PCOS and normal women, circulating levels of serum $\mathrm{LH}, \mathrm{FSH}, \mathrm{E}_{2}$, total testosterone (T), 17-hydroxy-progesterone (17-0HP), progesterone (P), insulin, and AMH were determined. Glucose levels were also assessed and insulin sensitivity calculated by quantitative insulin sensitivity check index (QUICKI) (13). Ovarian sonography was performed and ovarian size determined.

After the initial evaluation, the patients were followed without undergoing any specific treatment and were reassessed after 5 years, at a mean age was $42 \pm 2$ years. At this time, the mean age of the control subjects was $41 \pm 2$ years. After 5 years, 44 of the women with PCOS remained anovulatory and ten were ovulatory (ovulatory PCOS: ovulation-hyperandrogenism-polycystic ovaries). Menstrual patterns were recorded for $\geq 3$ months before the study and at follow-up; normal and irregular menses were defined as previously reported (see above).

Serum LH, FSH, E 2 , T, 17-OHP, DHEAS, and AMH were determined during the follicular phase (days 5-8) of a spontaneous or progestin-induced cycle. Serum $\mathrm{P}$ was determined on days 21-24 to determine ovulatory function. Serum insulin and blood glucose were measured in the fasting state.

Serum hormone levels were quantified by well established methods which had been validated previously in our laboratory. All steroids were measured by specific RIAs after extraction using previously described methods (14). In all assays, intra-assay and interassay coefficients of variation did not exceed 6\% and 15\%, respectively.

During the first evaluation, AMH was measured by a commercial ELISA provided by Diagnostics Systems Laboratory (DSL 10-14400). At the second evaluation, because of the consolidation of this company by Beckman Coulter, the Beckman assay was used (AMH Gen II assay). According to multicenter studies $(15,16)$, the GEN 11 assays result in values $\sim 30 \%-40 \%$ higher than the original DSL assay, but with similar precision and an excellent between-assay agreement. This conversion of $\mathrm{AMH}$ in $\mathrm{ng} / \mathrm{mL}$ to $\mathrm{pmol} / \mathrm{L}$ requires that values be multiplied by 7.143.

Anovulation was defined as serum $\mathrm{P}<3 \mathrm{ng} / \mathrm{mL}(<9.54$ $\mathrm{nmol} / \mathrm{L})$. In patients with normal menses, at least two consecutive menstrual cycles were studied, and the finding of low levels of serum $\mathrm{P}(<3 \mathrm{ng} / \mathrm{mL})$ in both cycles indicated the presence of chronic anovulation.

Biochemical hyperandrogenism was defined as serum $\mathrm{T}$ $>60 \mathrm{ng} / \mathrm{dL}(>2.08 \mathrm{nmol} / \mathrm{L})$ and/or serum DHEAS $\geq 3 \mu \mathrm{g} /$ $\mathrm{mL}(\geq 7.8 \mu \mathrm{mol} / \mathrm{L})$. These values for hyperandrogenism have been previously validated with the use of the previously described assays (17).

In all patients and control subjects, ovarian morphology was assessed by pelvic ultrasound. Ovarian size was determined, as was the presence, size, and number of ovarian follicles 2-10 $\mathrm{mm}$.

The presence of polycystic ovaries was established by the presence of ten or more peripherally oriented cystic structures in one ultrasonographic plane, each of which measured 2-10 $\mathrm{mm}$ in diameter, arranged around a dense stroma (17, 18). Ovarian volume was calculated by the formula $\pi / 6$ $(\mathrm{D} 1 \times \mathrm{D} 2 \times \mathrm{D} 3)$ where the dimensions (D) of length, width, and thickness were used. The size of both ovaries was assessed and mean ovarian size was calculated. According to our data in normal women, increased ovarian size was defined as a mean ovarian size $>7.5 \mathrm{~mL}$ (19).

The adopted procedures were in agreement with the Helsinki Declaration of 1975, and the study was approved by the local Ethics Council of Palermo University. All subjects gave their informed consents to participate in the study.

\section{Statistical Analysis}

Group means were compared with the use of analysis of variance with post hoc evaluation by the method of least squares and by pairwise means comparisons (after log transformation of the values). Pearson test was used for correlations. All data are presented as mean \pm SD.

\section{RESULTS}

At the initial evaluation (Table 1) women with PCOS had a body mass index (BMI; $28.2 \pm 3 \mathrm{~kg} / \mathrm{m}^{2}$ ) similar to, and levels of circulating LH, LH/FSH ratio, T, DHEAS, insulin, and AMH higher than matched control subjects of similar age. Insulin sensitivity calculated by QUICKI was lower. Women with PCOS also had increased ovarian volume (10.2 \pm 2.7 vs. $4.8 \pm 1.1 \mathrm{~mL} ; P<.01)$. The same parameters were still 


\section{TABLE 1}

Some clinical and endocrine parameters in 54 PCOS women and 20 control subjects aged 35-40 years and 5 years later.

$\begin{array}{lrrrrrrrrr} & \text { Age, } \mathbf{y} & \mathbf{B M I}, \mathbf{k g} / \mathbf{m}^{\mathbf{2}} & \mathbf{L H}, \mathbf{m U I} / \mathbf{m L} & \mathbf{L H} / \mathbf{F S H} \text { ratio } & \mathbf{T}, \mathbf{n g} / \mathbf{d L} & \mathbf{D H E A S}, \boldsymbol{\mu g} / \mathbf{m L} & \mathbf{A M H}, \mathbf{n g} / \mathbf{m L} & \mathbf{I R I}, \boldsymbol{\mu U} / \mathbf{m L} & \mathbf{Q U I C K I} \\ \text { PCOS } & 37 \pm 1 & 28 \pm 6 & 10 \pm 3.7 & 1.6 \pm 0.7 & 74 \pm 22 & 2.4 \pm 1 & 6.7 \pm 2.1 & 15.1 \pm 6.5 & 0.325 \pm 0.02 \\ \text { Control } & 37 \pm 1 & 27.4 \pm 3 & 6.4 \pm 1.4 & 1.1 \pm 0.2 & 28 \pm 11 & 1.8 \pm 1 & 1.7 \pm 0.7 & 9.4 \pm 2 & 0.355 \pm 0.02 \\ \text { PCOS } & 42 \pm 1 & 28 \pm 6 & 8.8 \pm 4 & 1.5 \pm 0.7 & 58 \pm 19 & 2.2 \pm 1.2 & 3.9 \pm 1.2 & 14 \pm 4 & 0.325 \pm 0.02 \\ \text { Control } & 42 \pm 1 & 28 \pm 4 & 6.5 \pm 1.1 & 1 \pm 0.3 & 25 \pm 16 & 1.7 \pm 1 & 1 \pm 0.7 & 9.5 \pm 2.4 & 0.351 \pm 0.02\end{array}$

Note: $\mathrm{AMH}=$ antimüllerian hormone; $\mathrm{BMI}=$ body mass index; IRI = immunoreactive insulin; PCOS = polycystic ovary syndrome; QUICKI = quantitative insulin sensitivity check index.

Carmina. AMH and normalization of menses in PCOS. Fertil Steril 2012.

significantly increased in PCOS women compared with control subjects when evaluated after 5 years (Table 1).

Comparing the values at the second evaluation with the initial levels ( 5 years before) in both PCOS and control subjects, a significant decrease of AMH was observed (Fig. 1), whereas the values of $\mathrm{T}$ and DHEAS decreased significantly $(P<.01)$ only in women with PCOS (data not shown). No significant changes in BMI, LH, LH/FSH ratio, insulin, QUICKI, and ovarian size were observed. Interestingly, the mean decrease in AMH was similar in women with PCOS $(40 \pm 12 \%)$ and control subjects $(41 \pm 10 \%)$.

The women with PCOS who were ovulatory after 5 years had lower serum LH, T, and AMH than the anovulatory group, but the differences were significant only for AMH values $(2.4 \pm 0.8$ vs. $4.3 \pm 0.90 \mathrm{ng} / \mathrm{mL} ; P<.01)$. However, the ovulatory group of women with PCOS still had higher AMH values compared with values in the normal aging control subjects $(1.0 \pm 0.7 \mathrm{ng} / \mathrm{mL} ; P<.01)$. Over the 5 years, there was no significant change in ovarian size, BMI, or insulin sensitivity.

Presence of ovulation (evaluated by P levels) after 5 years, at the mean age of 42 years, did not correlate with BMI or any initial hormonal parameter at the age of 37 years, with the exception of AMH values $(r=-0.42 ; P<.05)$, suggesting that a lower AMH level is associated with the chance of ovulatory function. In line with this, patients who became ovulatory with aging had significantly lower levels of AMH at their initial evaluation (anovulatory patients who became ovulatory: $4.8 \pm 1.7 \mathrm{ng} / \mathrm{mL}$; vs. anovulatory patients who remained

\section{FIGURE 1}

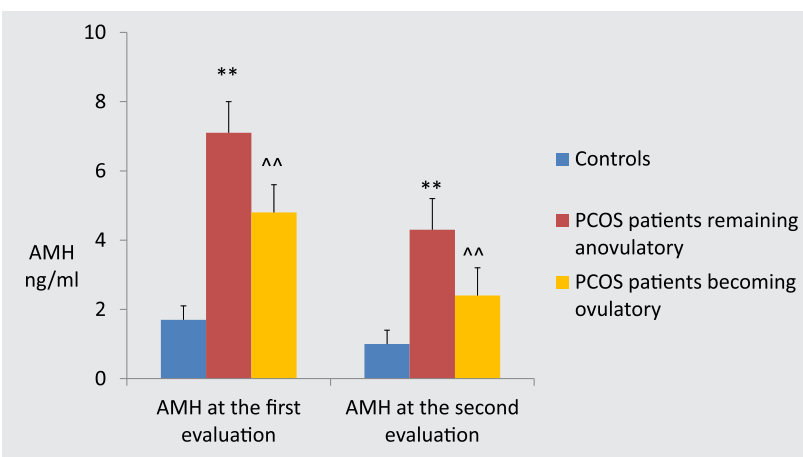

Bar graph of values of antimüllerian hormone $(\mathrm{AMH})$ before and 5 years after in anovulatory and ovulatory group and control subjects. PCOS $=$ polycystic ovary syndrome. ${ }^{* * P}<.01$ vs. control and PCOS becoming ovulatory. ${ }^{\wedge} P<.01$ vs. control.

Carmina. AMH and normalization of menses in PCOS. Fertil Steril 2012. anovulatory: $7.1 \pm 2 \mathrm{ng} / \mathrm{mL} ; P<.01)$. After 5 years, the ovulatory group had mean values of $2.4 \pm 0.8 \mathrm{ng} / \mathrm{mL}$, which remained significantly lower than the anovulatory group at the same time point: $4.3 \pm 0.9 \mathrm{ng} / \mathrm{mL}(P<.01$; Fig. 1$)$.

Evaluating absolute values, we did not find a clear predictive value of AMH for ovulatory function, but women with PCOS having AMH values $<5 \mathrm{ng} / \mathrm{mL}$ had a $60 \%$ probability to ovulate by age 42 , and all women having values $\leq 4 \mathrm{ng} / \mathrm{mL}$ had ovulatory function. None of the women who remained anovulatory had initial values of AMH $<4$ $\mathrm{ng} / \mathrm{mL}$. The complete range of values in the ovulatory group was $3.1-8.0 \mathrm{ng} / \mathrm{mL}$ initially and $1.8-3.3 \mathrm{ng} / \mathrm{mL}$ after 5 years. Corresponding complete ranges in the anovulatory group were $4.1-12 \mathrm{ng} / \mathrm{mL}$ initially and $2.9-7.3 \mathrm{ng} / \mathrm{mL}$ after 5 years.

\section{DISCUSSION}

In this study we assessed the endocrine profile of a group of women with PCOS women aged 35-40 years who were reevaluated after 5 years. The aim of this study was to understand whether any hormonal or ovarian parameter may be helpful in determining menstrual patterns and ovulatory function as the disorder evolves with aging. It has been described previously that with aging, women with PCOS show a significant improvement in their endocrine profiles and that this may be associated with the observation of more normal menses and ovulatory cycles (1-4). However, only a proportion of patients normalize their menses and resume ovulatory cycles.

In the present study, 10 anovulatory patients out of 54 $(\sim 20 \%)$ became ovulatory at a mean age of 42 years with a prevalence of improvement that was similar to what we observed previously (4). Here we showed that ovulation appeared in women with PCOS who had lower values of AMH values 5 years earlier. Although AMH levels correlated with serum $\mathrm{T}$ and insulin, no significant correlation was found between changes in those hormonal parameters and ovulatory function. Also in the 5 years of follow-up, we did not observe changes in ovarian size, BMI, or insulin sensitivity. Clearly we would expect to see such changes with a longer follow-up, as has been described previously (4). Although the present numbers are small, with only $20 \%$ of the women having ovulatory function at 5 years, the data suggest that a critical level of serum AMH is $\sim 4 \mathrm{ng} / \mathrm{mL}$. None of the group that remained anovulatory had values below that value at their initial evaluation. 
One of the potential concerns in these data is that the assays for AMH were carried out with reagents from different manufacturers, although we have been reassured that in this instance, the assays are the same, and this occurred merely due to a commercial acquisition. Moreover even if values are slightly higher or lower between the assays in the two time periods, it would have affected the subgroups in the same way; and although absolute values and cutoff points may not be precise, the trends, and therefore our conclusions, are expected not to be different. It should also be noted that in our previous study of a 20-year follow-up period (4), other parameters, such as $\mathrm{T}$, were observed to decrease, which was not the case here with only 5 years of observation. Our observations of excursions in AMH and menstrual function are more appropriate with the shorter follow-up, in that a longer follow-up puts many women into perimenopause or beyond.

It is well known that AMH levels are higher in women with PCOS compared with normal control subjects of similar age (20-24) and that among women with various phenotypes of PCOS, the anovulatory group of women have the highest AMH values (22). In addition, in PCOS, serum levels of AMH generally correlate with serum androgens and with insulin (21-23), which also relate to the more severe phenotypes. Finally, earlier studies have found that increased AMH levels in PCOS are not only correlated with the increased number of small preantral follicles, but also to some intrinsic abnormality of granulosa cell function (24). These findings may signify that in some way, AMH levels express the severity of the disorder probably reflecting the severity of derangement in folliculogenesis (24). Also, these data are similar but different from other data that in women undergoing laparoscopic ovarian diathermy, the women who became ovulatory had lower levels of serum AMH before treatment (25).

These data would be consistent with the view that the clinical improvement in women with PCOS is greater in those women with less compromised folliculogenesis and perhaps a smaller follicular cohort (11). Our prospective observation, although small, of lower serum AMH predicting more ovulatory function may provide some useful clinical information in counseling women with PCOS, and helps in our understanding of altered folliculogenesis in women with PCOS.

We conclude that although serum AMH decreases with age, values remain elevated compared with normal women, even in those women with PCOS who have ovulatory function. Women with PCOS who become ovulatory with aging have lower levels of serum AMH not only in the fourth decade, but also at a younger age. We hypothesize that the level of AMH possibly reflects the severity of the disordered folliculogenesis in women with PCOS, and that the occurrence of ovulation with aging in women with PCOS occurs in those women with a milder follicular derangement.

\section{REFERENCES}

1. Elting MW, Korsen TJ, Rekers-Mombarg LT, Schoemaker J. Women with polycystic ovary syndrome gain regular menstrual cycles when ageing. Hum Reprod 2000;15:24-8.
2. Liang SJ, Hsu CS, Tzeng CR, Chen CH, Hsu MI. Clinical and biochemical presentation of polycystic ovary syndrome in women between the ages of 20 and 40. Hum Reprod 2011;26:3443-9.

3. Brown ZA, Louwers YV, Fong SL, Valkenburg O, Birnie E, de Jong FH, et al. The phenotype of polycystic ovary syndrome ameliorates with aging. Fertil Steril 2011;96:1259-65.

4. Carmina E, Campagna AM, Lobo RA. 20 years follow-up of young PCOS women. Obstet Gynecol 2012;119:263-91.

5. Piltonen T, Koivunen R, Ruokonen A, Tapanainen JS. Ovarian age-related responsiveness to human chorionic gonadotropin. J Clin Endocrinol Metab 2003;88:3327-32.

6. Labrie F, Belanger A, Cusan L, Gomez JL, Candas B. Marked decline in serum concentrations of adrenal $\mathrm{C} 19$ sex steroid precursors and conjugated androgen metabolites during aging. J Clin Endocrinol Metab 1997;82:2386-92.

7. Pavlik EJ, de Priest PD, Gallion HH, Ueland FR, Reedy MB, Kryscio RJ, van Nagell JR Jr. Ovarian volume related to age. Gynecol Oncol 2000;77:410-2.

8. Oppermann K, Fuchs SC, Spritzer PM. Ovarian volume in pre- and perimenopausal women: a population-based study. Menopause 2003;10:209-13.

9. Winters SJ, Talbott E, Guzick DS, Zborowski J, McHugh KP. Serum testosterone levels decrease in middle age in women with the polycystic ovary syndrome. Fertil Steril 2000;73:724-9.

10. Moran C, Knochenhauer E, Boots LR, Azziz R. Adrenal androgen excess in hyperandrogenism: relation to age and body mass. Fertil Steril 1999;71:671-4.

11. Elting MW, Kwee J, Korsen TJM, Rekers-Mombarg LTM, Schoemaker J. Aging women with polycystic ovary syndrome who achieve regular menstrual cycles have a smaller follicle cohort than those who continue to have irregular cycles. Fertil Steril 2003;79:1154-60.

12. Rotterdam ESHRE/ASRM Sponsored PCOS Consensus Workshop Group. Revised 2003 consensus on diagnostic criteria and long-term health risks related to polycystic ovary syndrome. Fertil Steril 2004;81:19-25.

13. Katz A, Nambi SS, Mather K, Baron AD, Follmann DA, Sullivan G, Quon MJ. Quantitative insulin sensitivity check index: a simple, accurate method of assessing insulin sensitivity in humans. J Clin Endocrinol Metab 2000;85: 2402-10.

14. Wallace AM, Faye SA, Fleming R, Nelson SM. A multicentre evaluation of the new Beckman Coulter anti-mullerian hormone immunoassay (AMH Gen II). Ann Clin Biochem 2011;48:370-3.

15. Nelson SM, La Marca A. The journey from the old to the new AMH assay: how to avoid getting lost in the values. Reprod Biomed Online 2011;23: 411-20.

16. Carmina E. Prevalence of idiopathic hirsutism. Eur J Endocrinol 1998;139: $421-3$

17. Balen AH, Laven JS, Tan SL, Dewailly D. Ultrasound assessment of the polycystic ovary: international consensus definitions. Hum Reprod Update 2003; 9:505-14.

18. Carmina E, Orio F, Palomba S, Longo RA, Lombardi G, Lobo RA. Ovarian size and blood flow in women with polycystic ovary syndrome (PCOS) and their correlations with some endocrine parameters. Fertil Steril 2005;84:413-9.

19. Pigny $P$, Merlen E, Robert $Y$, Cortet-Rudelli C, Decanter C, Jonard S, Dewailly $D$. Elevated serum level of anti-müllerian hormone in patients with polycystic ovary syndrome: relationship to the ovarian follicle excess and to the follicle arrest. J Clin Endocrinol Metab 2003;88:5957-62.

20. Chu MC, Carmina E, Wang J, Lobo RA. Mullerian-inhibiting substance reflects ovarian findings in women with polycystic ovary syndrome better than does inhibin-B. Fertil Steril 2005;84:1685-8.

21. Pellatt $L$, Rice $S$, Mason HD. Anti-müllerian hormone and polycystic ovary syndrome: a mountain too high? Reproduction 2010;139:825-33.

22. La Marca A, Giulini S, Tirelli A, Bertucci E, Marsella T, Xella S, Volpe A. Antimüllerian hormone measurement on any day of the menstrual cycle strongly predicts ovarian response in assisted reproductive technology. Hum Reprod 2007;22:766-71.

23. Pellatt L, Hanna L, Brincat M, Galea R, Brain H, Whitehead S, Mason H. Granulosa cell production of anti-müllerian hormone is increased in polycystic ovaries. J Clin Endocrinol Metab 2007;92:240-5.

24. Amer SA, Li TC, Ledger WL. The value of measuring anti-mullerian hormone in women with anovulatory polycystic ovary syndrome undergoing laparoscopic ovarian diathermy. Hum Reprod 2009:24:2760-6. 